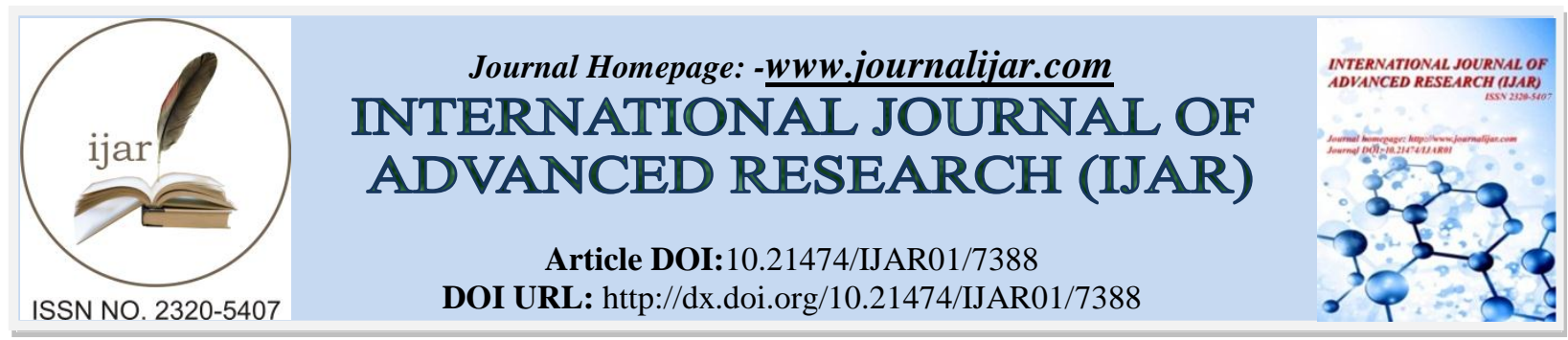

RESEARCH ARTICLE

\title{
CRANIOFACIALMORPHOLOGY OF 9 YEARS RURAL RESIDENTIAL SCHOOL CHILDREN OF SUTTUR.
}

Ravi Shanthraj ${ }^{1}$, Nandlal $B^{2}$, Karthikeya Patil ${ }^{3}$ and Ashwini $B^{4}$.

1. Associate Professor, Dept of Orthodontics, JSS Dental College and Hospital, Mysore, Karnataka, India.

2. Professor in Pedodontics and Preventive Dentistry, JSS Dental College and Hospital, Mysore, Karnataka, India.

3. Professor \& Head Dept. of Oral Medicine \& Radiology, JSS Dental College and Hospital, Mysore, Karnataka, India.

4. Reader, Conservative and Endodontics, Farooqia Dental College \& Hospital, Mysore.

\section{Manuscript Info}

Manuscript History

Received: 10 May 2018

Final Accepted: 12 June 2018

Published: July 2018

\section{Abstract}

Introduction: Most of the craniofacial growth studies are crosssectional in nature and provided a large number of cephalometric standards for males and females at different ages and for various ethnic groups. Variations in craniofacial Morphology exist between different ethnic and racial groups. The cephalometric standards developed for one ethnic group need not apply to other ethnic groups - the racial groups must be dealt according to their own characteristics. Limited number of longitudinal or semi-longitudinal studies has been reported for Indian population. Objective: The present study was designed to describe craniofacial morphology of rural residential Free school children of Suttur at 9 years. Material and Methods: 10 Males \& 10 Females of 9 years old children were selected and Lateral Cephalograms were taken. On each Lateral Cephalogram, 21 skeletal cephalometric landmarks were identified. From the cephalometric landmarks and reference lines, 31 angular \& linear measurements were analyzed, which were grouped as JSS Craniofacial analysis. Results \& Discussion: Mean Sagittal Measurements of 9 year old when compared between genders, there was no statistical significant difference. Females showed higher values than males for variables SNA, SNB, ANB, A-Npog, A:ss-n-pg. Mean vertical measurements when compared between genders for PFH, AFH \& N:ANS were found to be statistically significant and males had higher values than the females. Mean Mandibular length measurements Go-Co and Co-Gn when compared between genders males had higher Mean values for both parameters were was statistically significant. Mean Dental measurements when compared between genders for values of L1-APog, U1-NA, L1-NB, U1-NL, U1-Protrusion, U1-Inclination, L1-Inclination and U1-L1, there was no significant difference between genders for any variables. Males had higher Mean values than females for all caranial base measurements. The variables $\mathrm{S}: \mathrm{Ba} \& \mathrm{ACB}$ showed statistically significant difference between genders. Conclusion: Craniofacial Morphological data obtained in this study is very useful, but needs to be done on a larger sample. 


\section{Introduction:-}

Craniofacial growth is a complex and beautiful phenomenon. Growth has been described in so many terms. Todd defines growth as "increase in size". Meredith defines growth as the entire series of anatomic and physiologic changes taking place between the beginning of prenatal life and the close of senility. Growth is quantitative, i.e., it is measureable aspect of biologic life. ${ }^{1-3}$ Variations in craniofacial Morphology exist between different ethnic and racial groups. There is a lack of studies on cephalometric standard values for Indian School children. Thus the present study was designed to describe the Craniofacial morphology of rural residential Free school children of Suttur at 9 years.

\section{Materials and Methods:-}

The subjects were from residential free school of Suttur who had clinically acceptable occlusion having normal growth and no history of orthodontic treatment. Ethical Clearance was obtained from Institutional Ethical committee. Informed consent of parents or guardians was obtained for children.

Estimation of sample size was based on mean and standard deviation of parameters under study. In order to detect a Clinically Relevant Difference (CRD) at 5\% at $95 \%$ confidence and $90 \%$ power, required sample size was provisionally estimated to be 20 . In order to study changes in Cepholometric measurements 10 Male \& 10 Female of 9 years old children were selected and Lateral Cephalograms were taken using - Trophy Ortho Slice $1000 \mathrm{C}$ in Department of Oral Medicine \& Radiology of JSS Dental College and Hospital, with teeth in centric occlusion. Films were processed in Promax Automatic x-ray film processor. The Lateral Cephalograms were 492igitized using Epson V 700 dual bedded scanner at 720 dpi and tracing was done using Nemoceph studio software.

On each Lateral Cephalogram, 21 skeletal landmarks (Figure 1) were identified. From them 31 angular and linear measurements were derived, which were grouped as JSS Craniofacial analysis (JSS Growth analysis). These angular and linear measurements were taken from Steiner's ${ }^{4}$, Tweed's ${ }^{5}$, Down's ${ }^{6}$, Rickettes ${ }^{7}$, Rakosi Jarbak ${ }^{8}$, McNamara $^{9}$ and Bjork ${ }^{10}$ analysis. For purpose of convenience and better understanding findings of the study were grouped under five headings - 1) Basal Sagittal Measurements 2) Basal Vertical Measurements 3) Mandibular length measurements 4) Dental Measurements \& 5) Cranial Base Measurements.

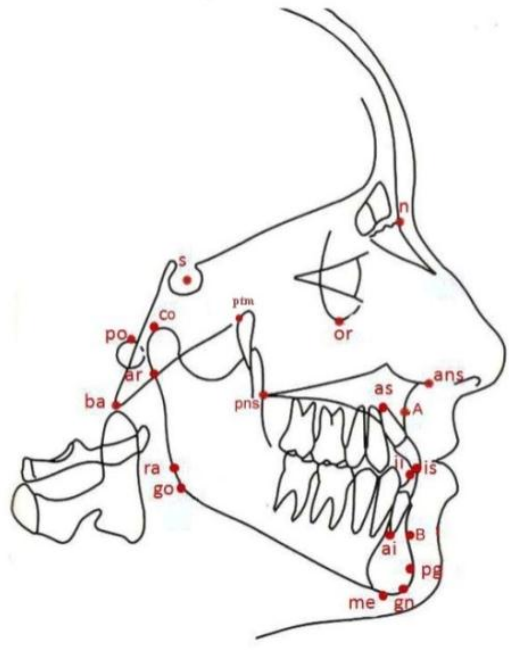

Fig 1:-Cephalometric landmarks

Linear and Angular Measurements:-

Basal Sagittal measurements:-

Angular: SNA, SNB, ANB, A:ss-n-Pg, S-N-Pg

Linear: A-NPog 


\section{Basal Vertical measurements:-}

Angular: Jaw relationship, Maxillary Inclination, Jaw Angle, Mandibular Inclination, SN-PoOr.

Linear: PFH, AFH, PFH/AFH, N:ans, Ans:Gn

\section{Mandibular Length:-}

Linear: Go-Co, Co-Gn

\section{Dental measurements:-}

Angular: U1-NL, U1- Inclination, L1-Inclination, U1-L1.

Linear: L1-APog, U1-NA, L1-NB, U1-Protrusion.

Cranial Base Measurements:-

Angular: n-s-ar, n-s-ba,

Linear: S:Ba, PCB, ACB.

\section{Statistical Analysis:-}

Descriptive and Inferential statistics were employed and level of statistical significance was predetermined at the 0.05 level of confidence. Descriptive statistics that included mean, S.D, Standard Error, 95\% confidence interval for mean, Minimum, Maximum values were calculated for each parameter. Inferential Statistics - Independent sample t test between Males and females were compared by using Independent student $t$ test. The Statistical analysis was done using SPS software version 20.

\section{Results and Discussion:-}

Craniofacial morphology and their variations amongst various ethnic groups has long been a subject of investigation. Establishing norms for different populations \& racial groups is very helpful in prediction, diagnosis and treatment planning. Many studies have concluded that standard measurements of one racial group cannot be considered for other racial groups. ${ }^{11,12}$ In the Indian scenario, the amount of cephalometric studies to establish norms for Indian school children are very scanty and hence have been using western norms which would not be relevant to the Indian population. ${ }^{13-15}$ Thus the purpose of this study was to establish lateral cephalometric craniofacial Morphology at 9 years, and also to evaluate possible differences between genders.

Table 1:-Basal Sagittal Measurements - at 9 Years of age.

\begin{tabular}{|c|c|c|c|c|c|c|c|c|c|c|c|}
\hline \multirow[t]{2}{*}{$\begin{array}{l}\text { Basal Sagittal } \\
\text { Measurement }\end{array}$} & \multirow[t]{2}{*}{$\begin{array}{l}\mathbf{N} \\
(20)\end{array}$} & \multirow[t]{2}{*}{ Mean } & \multirow[t]{2}{*}{$\overline{S \text { D }}$} & \multirow[t]{2}{*}{$\begin{array}{l}\text { Std. } \\
\text { Error }\end{array}$} & \multicolumn{2}{|c|}{$\begin{array}{l}95 \% \\
\text { Confidence } \\
\text { Interval for } \\
\text { Mean }\end{array}$} & \multirow[t]{2}{*}{ Min } & \multirow[t]{2}{*}{ Max } & \multicolumn{3}{|c|}{$\begin{array}{l}\text { Comparison } b / w \\
\text { males and females }\end{array}$} \\
\hline & & & & & $\begin{array}{l}\text { Lower } \\
\text { Bound }\end{array}$ & $\begin{array}{l}\text { Upper } \\
\text { Bound }\end{array}$ & & & $\begin{array}{l}\text { Mean } \\
\text { Diff. }\end{array}$ & $\begin{array}{l}\mathbf{t} \\
\text { value }\end{array}$ & $\begin{array}{l}\mathbf{p} \\
\text { value }\end{array}$ \\
\hline \multirow[t]{2}{*}{ SNA } & $\mathrm{M}$ & 79.85 & 1.20 & 0.38 & 78.99 & 80.71 & 78.90 & 82.30 & \multirow[t]{2}{*}{0.26} & \multirow[t]{2}{*}{-0.566} & \multirow[t]{2}{*}{0.578} \\
\hline & $\mathrm{F}$ & 80.11 & 0.82 & 0.26 & 79.52 & 80.70 & 79.00 & 81.70 & & & \\
\hline \multirow[t]{2}{*}{ SNB } & M & 77.77 & 1.99 & 0.63 & 76.35 & 79.19 & 75.00 & 82.20 & \multirow[t]{2}{*}{0.5} & \multirow[t]{2}{*}{0.538} & \multirow[t]{2}{*}{0.597} \\
\hline & $\mathrm{F}$ & 77.27 & 2.16 & 0.68 & 75.73 & 78.81 & 73.30 & 80.10 & & & \\
\hline \multirow[t]{2}{*}{ ANB } & M & 2.09 & 1.37 & 0.43 & 1.11 & 3.07 & 0.10 & 4.20 & \multirow[t]{2}{*}{0.76} & \multirow[t]{2}{*}{-1.117} & \multirow[t]{2}{*}{0.279} \\
\hline & $\mathrm{F}$ & 2.85 & 1.66 & 0.53 & 1.66 & 4.04 & 0.70 & 5.70 & & & \\
\hline \multirow[t]{2}{*}{ A-NPog } & $\mathrm{M}$ & 2.05 & 2.11 & 0.67 & 0.54 & 3.56 & -1.90 & 4.70 & \multirow[t]{2}{*}{0.85} & \multirow[t]{2}{*}{\begin{tabular}{|l|}
-0.99 \\
\end{tabular}} & \multirow[t]{2}{*}{0.335} \\
\hline & $\mathrm{F}$ & 2.90 & 1.71 & 0.54 & 1.67 & 4.13 & 0.30 & 5.90 & & & \\
\hline \multirow[t]{2}{*}{ A:sS-n-pg } & $\mathrm{M}$ & 2.33 & 2.51 & 0.79 & 0.53 & 4.13 & -2.60 & 5.30 & \multirow[t]{2}{*}{1.29} & \multirow[t]{2}{*}{-1.253} & \multirow[t]{2}{*}{0.226} \\
\hline & $F$ & 3.62 & 2.07 & 0.66 & 2.14 & 5.10 & 0.40 & 6.90 & & & \\
\hline \multirow[t]{2}{*}{ s-n-pg } & $\mathrm{M}$ & 77.53 & 3.17 & 1.00 & 75.26 & 79.80 & 73.90 & 85.00 & \multirow[t]{2}{*}{1.06} & \multirow[t]{2}{*}{0.81} & \multirow[t]{2}{*}{0.428} \\
\hline & $F$ & 76.47 & 2.66 & 0.84 & 74.57 & 78.37 & 72.10 & 80.40 & & & \\
\hline
\end{tabular}

Results of Table 1 inferred that Mean Sagittal Measurements of 9 year old, when compared between males and females, there was no statistical significant difference between genders. Females showed higher values than males 
for variables SNA, SNB, ANB, A-Npog, A:ss-n-pg. But mean difference for variables was very small between genders.

Mean values of SNA for males was $79.85 \pm 1.20$ and for females was $80.11 \pm 0.82$, SNB for males was $77.77 \pm 1.99$ for females was $77.27 \pm 2.16$. Mean value of ANB for males was $2.09 \pm 1.37$ and females were $2.85 \pm 1.66$. These values suggested that though females had higher values for SNA and SNB the value of ANB should have decreased. But ANB also had higher value suggesting that females had retrognathic mandible than males. This was supported by mean value of s-n-pg which was $77.53 \pm 3.17$ for males and $76.47 \pm 2.66$ for females. Our study values were in agreement with studies of Savadi $\mathrm{S} \mathrm{C}^{17}$, but our values were closely matching with Chandranee ${ }^{16}$ study. Savadi.S.C. ${ }^{17}$ recorded ANB to be 5.75 for 6-9 years age group and 4.35 for $10-13$ years age group.

Table 2:-Basal Vertical Measurements - at 9 Years of age.

\begin{tabular}{|c|c|c|c|c|c|c|c|c|c|c|c|}
\hline \multirow[t]{2}{*}{$\begin{array}{l}\text { Basal vertical } \\
\text { Measurement }\end{array}$} & \multirow[t]{2}{*}{$\begin{array}{l}\mathbf{N} \\
(20)\end{array}$} & \multirow[t]{2}{*}{ Mean } & \multirow[t]{2}{*}{ S D } & \multirow[t]{2}{*}{$\begin{array}{l}\text { Std. } \\
\text { Error }\end{array}$} & \multicolumn{2}{|c|}{$\begin{array}{l}95 \% \\
\text { Confidence } \\
\text { Interval for } \\
\text { Mean }\end{array}$} & \multirow[t]{2}{*}{ Min } & \multirow[t]{2}{*}{ Max } & \multicolumn{3}{|c|}{$\begin{array}{l}\text { Comparison } b / w \\
\text { males and females }\end{array}$} \\
\hline & & & & & $\begin{array}{l}\text { Lower } \\
\text { Bound }\end{array}$ & $\begin{array}{l}\text { Upper } \\
\text { Bound }\end{array}$ & & & $\begin{array}{l}\text { Mean } \\
\text { Diff. }\end{array}$ & $\begin{array}{l}\mathbf{t} \\
\text { value }\end{array}$ & $\begin{array}{l}\mathbf{p} \\
\text { value }\end{array}$ \\
\hline \multirow[t]{2}{*}{ PFH } & $\mathrm{M}$ & 64.82 & 3.98 & 1.26 & 61.97 & 67.67 & 55.70 & 69.30 & \multirow[t]{2}{*}{3.17} & \multirow[t]{2}{*}{1.704} & \multirow[t]{2}{*}{0.106} \\
\hline & $\mathrm{F}$ & 61.65 & 4.33 & 1.37 & 58.55 & 64.75 & 53.10 & 68.90 & & & \\
\hline \multirow[t]{2}{*}{ AFH } & $\mathrm{M}$ & 98.70 & 5.51 & 1.74 & 94.76 & 102.64 & 90.30 & 107.30 & \multirow[t]{2}{*}{4.98} & \multirow[t]{2}{*}{2.373} & \multirow[t]{2}{*}{$0.029 *$} \\
\hline & $\mathrm{F}$ & 93.72 & 3.70 & 1.17 & 91.08 & 96.37 & 86.70 & 101.00 & & & \\
\hline \multirow[t]{2}{*}{ PFH/AFH } & M & 65.71 & 4.10 & 1.30 & 62.78 & 68.64 & 58.30 & 71.80 & \multirow[t]{2}{*}{0.03} & \multirow[t]{2}{*}{-0.016} & \multirow[t]{2}{*}{0.987} \\
\hline & $\mathrm{F}$ & 65.74 & 4.13 & 1.31 & 62.78 & 68.70 & 61.20 & 73.40 & & & \\
\hline \multirow[t]{2}{*}{ Jaw relation } & $\mathrm{M}$ & 24.72 & 4.69 & 1.48 & 21.37 & 28.07 & 18.90 & 32.50 & \multirow[t]{2}{*}{0.53} & \multirow[t]{2}{*}{-0.273} & \multirow[t]{2}{*}{0.788} \\
\hline & $\mathrm{F}$ & 25.25 & 3.95 & 1.25 & 22.42 & 28.08 & 18.70 & 29.00 & & & \\
\hline \multirow[t]{2}{*}{ Max Inclina } & $\mathrm{M}$ & 9.24 & 2.84 & 0.90 & 7.21 & 11.27 & 5.40 & 13.40 & \multirow[t]{2}{*}{0.7} & \multirow[t]{2}{*}{0.629} & \multirow[t]{2}{*}{0.537} \\
\hline & $\mathrm{F}$ & 8.54 & 2.08 & 0.66 & 7.05 & 10.03 & 5.30 & 12.20 & & & \\
\hline \multirow[t]{2}{*}{ Jaw angle } & $\mathrm{M}$ & 125.79 & 7.10 & 2.24 & 120.71 & 130.87 & 112.70 & 134.40 & \multirow[t]{2}{*}{0.05} & \multirow[t]{2}{*}{-0.018} & 0.986 \\
\hline & $\mathrm{F}$ & 125.84 & 5.42 & 1.71 & 121.96 & 129.72 & 115.50 & 135.20 & & & \\
\hline Mand .incli & $\mathrm{M}$ & 33.94 & 6.42 & 2.03 & 29.35 & 38.53 & 24.30 & 43.80 & 0.14 & 0.056 & 0.956 \\
\hline & $\mathrm{F}$ & 33.80 & 4.74 & 1.50 & 30.41 & 37.19 & 24.00 & 38.30 & & & \\
\hline SN-POOR & $\mathrm{M}$ & 8.15 & 1.01 & 0.32 & 7.43 & 8.87 & 6.90 & 10.00 & 0.22 & -0.507 & 0.619 \\
\hline & $\mathrm{F}$ & 8.37 & 0.93 & 0.29 & 7.71 & 9.03 & 7.00 & 9.40 & & & \\
\hline N:ANS & $\mathrm{M}$ & 44.52 & 4.18 & 1.32 & 41.53 & 47.51 & 38.00 & 52.00 & 2.31 & 1.654 & 0.115 \\
\hline & $\mathrm{F}$ & 42.21 & 1.42 & 0.45 & 41.20 & 43.22 & 40.10 & 44.60 & & & \\
\hline Ans: Gn & $\mathrm{M}$ & 53.14 & 2.04 & 0.64 & 51.68 & 54.60 & 50.60 & 56.70 & 2.48 & 2.281 & $0.035^{*}$ \\
\hline & $\mathrm{F}$ & 50.66 & 2.77 & 0.88 & 48.68 & 52.64 & 46.60 & 55.80 & & & \\
\hline
\end{tabular}

The results of table 2 inferred that Mean vertical measurements of 9 years old when compared between genders for AFH \&ANS:Gn were found to be satistically significant and males had higher values than the females. This Observation suggested that boys have relatively increased Anterior Facial Height and ANS:Gn.

The Mean values of males were higher than the females for PFH, AFH, Maxillary Inclination, Jaw Angle, N:ANS \&ANS:Gn also whereas the females showed Higher values for Jaw relation and SN-PoOr. Mean Mandibular Inclination in a study by Chandranee ${ }^{16}$ on age group of $4-5$ years was found to be $32.03 \pm 3.64$ and by Savadi.S.C ${ }^{17}$ on age group of 6-9 years was found to 34.75 and 29.9 at $10-13$ years and in our study it was found to be $33.94 \pm 6.42$ for males and $33.80 \pm 4.74$ for females at 9 years.

Table 3:-Mandibular length Measurements - at 9 Years of age.

\begin{tabular}{|l|l|l|l|l|l|l|l|l|}
\hline $\begin{array}{l}\text { Mandibular } \\
\text { length } \\
\text { Measurement }\end{array}$ & $\begin{array}{l}\mathbf{N} \\
\mathbf{( 2 0})\end{array}$ & Mean & S D & $\begin{array}{l}\text { Std. } \\
\text { Error }\end{array}$ & $\begin{array}{l}\text { 95\% } \\
\text { Confidence } \\
\text { Interval for } \\
\text { Mean }\end{array}$ & Min & Max & $\begin{array}{l}\text { Comparison b/w } \\
\text { males and females }\end{array}$ \\
\hline
\end{tabular}




\begin{tabular}{|l|l|l|l|l|l|l|l|l|l|l|l|}
\hline & & & & & $\begin{array}{l}\text { Lower } \\
\text { Bound }\end{array}$ & $\begin{array}{l}\text { Upper } \\
\text { Bound }\end{array}$ & & & $\begin{array}{l}\text { Mean } \\
\text { Diff. }\end{array}$ & $\begin{array}{l}\text { t } \\
\text { value }\end{array}$ & $\begin{array}{l}\text { p } \\
\text { value }\end{array}$ \\
\hline \multirow{2}{*}{ Go-Co } & M & 46.14 & 2.86 & 0.91 & 44.09 & 48.19 & 39.80 & 49.20 & 1.89 & 1.463 & 0.161 \\
\cline { 2 - 11 } & F & 44.25 & 2.91 & 0.92 & 42.17 & 46.33 & 38.90 & 48.80 & & & \\
\hline \multirow{2}{*}{ Co-Gn } & M & 93.23 & 4.77 & 1.51 & 89.81 & 96.65 & 85.10 & 100.20 & 4.45 & 2.559 & $0.02 *$ \\
\cline { 2 - 10 } & F & 88.78 & 2.73 & 0.86 & 86.83 & 90.73 & 83.10 & 92.50 & & & \\
\hline
\end{tabular}

$*_{p}<0.05$ and $* * p<0.01 ;$ Significant $* * * p<0.001$ Highly Significant; $p>0.05$ not significant

The results of Table 3 Inferred that mean Mandibular length measurements Go-Co and Co-Gn of 9 year old when compared between males and females, the males had higher Mean values than females for both parameters and was statistically significant when compared between genders. The above changes in 9 years of variables Go-Co and CoGn suggest that Males had increased growth in ramus height and length of Mandible, when compared with females. This suggests that males had a prominent mandible than females at 9 years.

Table 4:-Dental Measurements - at 9 Years of age.

\begin{tabular}{|c|c|c|c|c|c|c|c|c|c|c|c|}
\hline \multirow[t]{2}{*}{$\begin{array}{l}\text { Dental } \\
\text { Measurement }\end{array}$} & \multirow[t]{2}{*}{$\begin{array}{l}\mathbf{N} \\
(20)\end{array}$} & \multirow[t]{2}{*}{ Mean } & \multirow[t]{2}{*}{ S D } & \multirow[t]{2}{*}{$\begin{array}{l}\text { Std. } \\
\text { Error }\end{array}$} & \multicolumn{2}{|c|}{$\begin{array}{l}95 \% \\
\text { Confidence } \\
\text { Interval for } \\
\text { Mean } \\
\end{array}$} & \multirow[t]{2}{*}{ Min } & \multirow[t]{2}{*}{ Max } & \multicolumn{3}{|c|}{$\begin{array}{l}\text { Comparison } b / w \\
\text { males and females }\end{array}$} \\
\hline & & & & & $\begin{array}{l}\text { Lower } \\
\text { Bound }\end{array}$ & $\begin{array}{l}\text { Upper } \\
\text { Bound }\end{array}$ & & & $\begin{array}{l}\text { Mean } \\
\text { Diff. }\end{array}$ & $\begin{array}{l}\mathbf{t} \\
\text { value }\end{array}$ & $\begin{array}{l}\mathbf{p} \\
\text { value }\end{array}$ \\
\hline \multirow[t]{2}{*}{ L1-APog } & $\mathrm{M}$ & 4.02 & 2.60 & 0.82 & 2.16 & 5.88 & -0.90 & 6.80 & \multirow[t]{2}{*}{0.15} & \multirow{2}{*}{0.146} & \multirow{2}{*}{0.885} \\
\hline & $\mathrm{F}$ & 3.87 & 1.94 & 0.61 & 2.49 & 5.25 & 0.80 & 7.40 & & & \\
\hline \multirow[t]{2}{*}{ U1-Na } & $\mathrm{M}$ & 5.44 & 2.07 & 0.66 & 3.96 & 6.92 & 1.80 & 8.50 & \multirow[t]{2}{*}{0.77} & \multirow[t]{2}{*}{0.811} & \multirow[t]{2}{*}{0.428} \\
\hline & $\mathrm{F}$ & 4.67 & 2.17 & 0.69 & 3.12 & 6.22 & 1.20 & 7.60 & & & \\
\hline \multirow[t]{2}{*}{ L1-NB } & $\mathrm{M}$ & 4.91 & 2.14 & 0.68 & 3.38 & 6.44 & 1.00 & 7.90 & \multirow[t]{2}{*}{0.14} & \multirow[t]{2}{*}{0.182} & \multirow[t]{2}{*}{0.858} \\
\hline & $\mathrm{F}$ & 4.77 & 1.16 & 0.37 & 3.94 & 5.60 & 3.10 & 6.50 & & & \\
\hline \multirow[t]{2}{*}{ U1-NL } & $\mathrm{M}$ & 116.37 & 6.63 & 2.10 & 111.63 & 121.11 & 102.20 & 122.20 & \multirow[t]{2}{*}{2.26} & \multirow[t]{2}{*}{0.863} & \multirow[t]{2}{*}{0.4} \\
\hline & $\mathrm{F}$ & 114.11 & 4.97 & 1.57 & 110.55 & 117.67 & 106.30 & 120.50 & & & \\
\hline \multirow{2}{*}{$\begin{array}{l}\text { U1- } \\
\text { Protrusion }\end{array}$} & M & 7.07 & 2.22 & 0.70 & 5.48 & 8.66 & 2.60 & 9.20 & \multirow[t]{2}{*}{0.02} & \multirow[t]{2}{*}{0.023} & \multirow[t]{2}{*}{0.982} \\
\hline & $\mathrm{F}$ & 7.05 & 1.55 & 0.49 & 5.94 & 8.16 & 4.40 & 10.00 & & & \\
\hline \multirow{2}{*}{$\begin{array}{l}\text { U1- } \\
\text { Inclination }\end{array}$} & $\mathrm{M}$ & 107.13 & 6.43 & 2.03 & 102.53 & 111.73 & 95.50 & 114.40 & \multirow[t]{2}{*}{0.55} & \multirow[t]{2}{*}{0.189} & \multirow[t]{2}{*}{0.852} \\
\hline & $\mathrm{F}$ & 106.58 & 6.59 & 2.08 & 101.87 & 111.29 & 95.20 & 115.40 & & & \\
\hline \multirow{2}{*}{$\begin{array}{l}\text { L1 } \\
\text { Inclination }\end{array}$} & $\mathrm{M}$ & 101.07 & 6.74 & 2.13 & 96.25 & 105.89 & 88.00 & 112.70 & 3.27 & -1.143 & 0.268 \\
\hline & $\mathrm{F}$ & 104.34 & 6.03 & 1.91 & 100.02 & 108.66 & 93.50 & 112.60 & & & \\
\hline U1-L1 & $\mathrm{M}$ & 119.01 & 11.45 & 3.62 & 110.82 & 127.20 & 105.30 & 139.00 & 0.5 & 0.105 & 0.918 \\
\hline & $\mathrm{F}$ & 118.51 & 9.79 & 3.10 & 111.51 & 125.51 & 103.50 & 136.70 & & & \\
\hline
\end{tabular}

${ }^{*} p<0.05$ and ${ }^{* *} p<0.01 ;$ Significant $* * * p<0.001$ Highly Significant; $p>0.05$ not significant

The results of Table 4 Inferred that Mean Dental measurements of 9 year old when compared between Males and females for values of L1-APog, U1-NA, L1-NB, U1-NL, U1-Protrusion, U1-Inclination, L1-Inclination and U1-L1, there was differences in the mean values but it was not a statistically significant difference between genders for any variables. The Mean values of L1-APog, U1-NA, L1-NB, U1-NL, U1-Protrusion, U1-Inclination \& U1-L1 showed higher values for males than females, whereas females had higher mean values than males for L1-Inclination.

Mean U1-NA at 9 years for males was $5.44 \pm 2.07$ and for females was $4.67 \pm 2.17$ in our study, where as in the studies of Chandranee ${ }^{16}$ It was found to be $0.14 \pm 1.71$ for $4-5$ years of age group for Chandigar population. Savadi.S.C. ${ }^{17}$ found U1-NA to be $2.15 \pm 5.3$ for age group of 6-9 years of Karnataka population. The study of Savadi agreed with our study whereas the study of Chandranee was not in agreement because the age of the subjects was very less i.e. 4-5 years. The Mean L1-NB at 9 years for males was $4.91 \pm 2.14$ and for females was $4.77 \pm 1.16$ where as in studies of Savadi S.C. ${ }^{17}$ L1-NB was found to be $4.85 \pm 6.0$ for he age group of 6-9 years of Karnataka population, and study by Chandranee ${ }^{16}$ found L1-NB to be $2.80 \pm 1.31$ for age group of 4-5 years on Chandigarh population. L1 inclination in our study at 9 years for males was $101.07 \pm 6.74$ for females was $104.34 \pm 6.03$, whereas in study by Savadi S.C ${ }^{17}$ on 6-9 years found to be 93.65 and at 10-13years to be 100.00 for Karnataka population, this suggested that L1-Inclination was influenced by age and was in agreement with Savadi S C study. U1-L1 in our 
study, at 9 years was found to be $119.01 \pm 11.45$ for males and for females was $118.51 \pm 9.79$ Savadi S.C. ${ }^{17}$ in their study for age group of 6-9 years of Karnataka population found U1-L1 to be 131.9 was not matching with our study. D.N.Kapoor in a study on Lucknow Population of 6 years was $138.5 \pm 9.85$ and at 9 years was $125.33 \pm 7.49$.

Table 5:-Cranial base Measurements - at 9 Years of age.

\begin{tabular}{|c|c|c|c|c|c|c|c|c|c|c|c|}
\hline \multirow[t]{2}{*}{$\begin{array}{l}\text { Cranial base } \\
\text { Measurement }\end{array}$} & \multirow[t]{2}{*}{$\begin{array}{l}\mathbf{N} \\
(20)\end{array}$} & \multirow[t]{2}{*}{ Mean } & \multirow[t]{2}{*}{ S D } & \multirow[t]{2}{*}{$\begin{array}{l}\text { Std. } \\
\text { Error }\end{array}$} & \multicolumn{2}{|c|}{$\begin{array}{l}95 \% \\
\text { Confidence } \\
\text { Interval for } \\
\text { Mean } \\
\end{array}$} & \multirow[t]{2}{*}{ Min } & \multirow[t]{2}{*}{ Max } & \multicolumn{3}{|c|}{$\begin{array}{l}\text { Comparison } b / w \text { males } \\
\text { and females }\end{array}$} \\
\hline & & & & & $\begin{array}{l}\text { Lower } \\
\text { Bound }\end{array}$ & $\begin{array}{l}\text { Upper } \\
\text { Bound }\end{array}$ & & & $\begin{array}{l}\text { Mean } \\
\text { Diff. }\end{array}$ & $\begin{array}{l}\mathbf{t} \\
\text { value }\end{array}$ & p value \\
\hline \multirow[t]{2}{*}{ S:Ba } & $\mathrm{M}$ & 39.66 & 2.09 & 0.66 & 38.17 & 41.15 & 36.20 & 42.80 & \multirow[t]{2}{*}{2.74} & \multirow[t]{2}{*}{3.356} & \multirow[t]{2}{*}{$0.004 * *$} \\
\hline & $\mathrm{F}$ & 36.92 & 1.52 & 0.48 & 35.83 & 38.01 & 34.40 & 39.20 & & & \\
\hline \multirow[t]{2}{*}{ n-s-ar } & $\mathrm{M}$ & 121.89 & 3.75 & 1.19 & 119.21 & 124.57 & 116.90 & 127.40 & \multirow[t]{2}{*}{1.71} & \multirow[t]{2}{*}{-1.001} & \multirow[t]{2}{*}{0.33} \\
\hline & $\mathrm{F}$ & 123.60 & 3.89 & 1.23 & 120.82 & 126.38 & 115.60 & 127.70 & & & \\
\hline \multirow{2}{*}{ n-s-ba } & $\mathrm{M}$ & 129.80 & 3.50 & 1.11 & 127.30 & 132.30 & 123.90 & 134.80 & \multirow[t]{2}{*}{0.76} & \multirow[t]{2}{*}{-0.414} & \multirow[t]{2}{*}{0.684} \\
\hline & $\mathrm{F}$ & 130.56 & 4.63 & 1.46 & 127.25 & 133.87 & 122.20 & 136.50 & & & \\
\hline \multirow[t]{2}{*}{ PCB } & $\mathrm{M}$ & 28.57 & 1.87 & 0.59 & 27.23 & 29.91 & 26.00 & 31.30 & \multirow[t]{2}{*}{0.89} & \multirow[t]{2}{*}{0.999} & \multirow[t]{2}{*}{0.331} \\
\hline & $\mathrm{F}$ & 27.68 & 2.11 & 0.67 & 26.17 & 29.19 & 24.20 & 30.00 & & & \\
\hline \multirow[t]{2}{*}{ ACB } & $\mathrm{M}$ & 63.36 & 3.13 & 0.99 & 61.12 & 65.60 & 58.90 & 68.80 & \multirow[t]{2}{*}{2.25} & \multirow[t]{2}{*}{1.789} & \multirow[t]{2}{*}{$0.09 *$} \\
\hline & $\mathrm{F}$ & 61.11 & 2.45 & 0.77 & 59.36 & 62.86 & 58.40 & 65.90 & & & \\
\hline
\end{tabular}

Mean cranial base measurements of 9 year old when compared between Males and females, the males had higher Mean values than the females for all caranial base measurements. The variable S:Ba\& ACB showed statistically significant difference between the genders. The mean S:Ba at 9 years for Males was 39.66 \pm 2.09 and for females was $36.92 \pm 1.52$ the mean difference was 2.74 which was statistically significant. The mean ACB at 9 years for Males was $63.36 \pm 3.13$ and for females was $61.11 \pm 2.45$ the mean difference was 2.25 which were statistically significant. O.P.Kharabanda ${ }^{18} \&$ Ashima Valiathan ${ }^{19}$ in their study suggested that gender did not have any influence on the variable n-s-ar and this was matching with our study.

\section{Conclusion:-}

Craniofacial dimensions obtained in this study is very useful data of Craniofacial changes of Rural Residential School children of Suttur, but needs to be done on a larger sample.

\section{Bibliography:-}

1. Moyers, RE. Hand Book of Orthodontics, $4^{\text {th }}$ edition. Chicago: Year Book Medical Publishers Inc; 1988.

2. Enlow, Donal H. Essentials of Facial Growth, $2^{\text {nd }}$ edition, Philadelphia: Saunders c1996.

3. Sridhar Premkumar, Text Book of Craniofacial Growth, $1^{\text {st }}$ edition Jaypee Medical publishers 2011.

4. Steiner CC. Cephalometrics for you and me. Am J Orthod. 1953;39:729-755.

5. Tweed $\mathrm{CH}$. The Frankfort-mandibular incisor angle (FMIA) in orthodontic diagnosis, treatment planning and prognosis. Angle Orthod. 1954;24:121-169.

6. Downs WB. Analysis of the dentofacial profile. Angle Orthod 1956;26:191.

7. Ricketts RM. Perspectives in the clinical application of cephalometrics. Angle Orthod 1981;51:115.

8. Rakosi T. An Atlas and Manual of CephalometricRadiogrpahy. $1^{\text {st }}$ edition Wolfe Medical Publications Ltd. 1982.

9. McNamara Ja Jr. A method of cephalometric evolution. Am J Orthod 1984;86:449-469.

10. Bjork A, Skielles V. Facial development and tooth eruption. Am J Orthod 1972; 62:339-383.

11. Samir E Bishar, Longitudinal cephalometric standards from 5 years of age to adulthood. Am J Orthod;1981:3544.

12. Joseph E. Jamison, Samir E.Bishara, Larry C.Peterson, Charles R.Kremenak. Longitudinal changes in the maxilla and the maxillary-mandibular relationship between 8 and 17 years of age. Am J Orthod; 1982:217-230.

13. Srivats B, Nandlal B. Dento-Alveolar development and Craniofacial morphology in children with speech and hearing impairment. MDS Thesis, RGUHS, Bangalore Sept 2000. 
14. Agastayya C, Nandlal B. Maxillary dento-alveolar development in unilateral cleft lip and palate cases after cheiloplasty - A Retrospective evaluation. MDS Thesis, RGUHS, Bangalore March 2001.

15. Prashanth S, Nandlal B. Craniofacial and dentoalveolar morphological changes in Asthamtic Children - A cephalometric study. MDS Thesis, RGUHS, Bangalore April 2007.

16. Chandranee. RoentgenographicCephalometric study of North Indian children and Permanent Dentition. MDS Thesis, PGI, Chandigarh 1982.

17. Jyothindra Kumar K. A hand book of Cephalometric norms for Indian ethnic groups.1999 printed and published by Indian Orthodontic Society.

18. Kharbanda OP, Sindhu SS, Sundram KR. Cephalometric profile of Aryodravidians - I and II. Study of denture bases. J Indian Orthod Soc. 1989;20:84-94.

19. Manish Valithan, AshimaValithan and V Ravinder .JarabakCephalometric Analysis Rebron. J Ind. OrthodSoc 2001; 34:66-76.

20. Nandlal B, KarthikeyaPatil\& Ravi S Craniofacial Changes in Rural Residential School Children of Suttur of 7 years of Age. International Journal of Dental Science-IJDE 1.1(2015): 15-23. 